\title{
Perceptual asynchronies between color and motion at the onset of motion and along the motion trajectory
}

\author{
Angélique Gauch ANd Dirk Kerzel \\ University of Geneva, Geneva, Switzerland
}

\begin{abstract}
A color change that is physically simultaneous with the onset of object motion may be perceived as occurring before the initial displacement. In contrast, a colored flash during object motion is displaced in the direction of motion, suggesting that it is perceived after the continuous position change. The aim of our study was to reconcile these apparently conflicting results. To this end, we reexamined color-motion asynchronies as a function of trajectory position. Our results indicate that an abrupt color change lags object motion along the trajectory, but no asynchrony was found when the abrupt color change occurred at motion onset. Even if the lag of color relative to motion decreased with increasing object size, we did not replicate a lag of motion relative to color in any of our experiments. Furthermore, judgments at motion onset were not correlated with judgments along the trajectory, suggesting that the underlying mechanisms or task demands were different. Temporal order may be judged at motion onset, whereas position is judged during ongoing motion.
\end{abstract}

Anatomical and physiological studies have demonstrated that motion and color are processed in separate cortical areas. Motion is processed in area MT in the magnocellular pathway, and color is processed in area V4 in the parvocellular pathway (e.g., Livingstone \& Hubel, 1988). These findings led to a modular view of vision, which considers each brain site to be a processing system that acts independently and in parallel with other systems. Evidence for cortical areas that specialize in processing particular attributes of a visual scene poses a fundamental problem for visual perception. The question is this: How we can perceive a unitary and coherent visual scene when attributes are processed in different brain regions? This problem is known as the binding problem, and different mechanisms have been suggested for explaining how the brain binds cortical activity in order to create a unified percept (Engel, Fries, König, Brecht, \& Singer, 1999; Treisman, 1999).

In this context, Moutoussis and Zeki (1997a, 1997b) proposed a new account involving a binding process, not of the cortical activity, but of different microconsciences generated by distinct cortical areas. They suggested that particular modules (e.g., MT, V4) produce distinct perceptual experiences of their preferred attributes, meaning that different microconsciences must be bound. On the basis of the assumption that processing times are probably not the same in the different modules, Moutoussis and Zeki (1997a, 1997b) hypothesized that it would be likely that different attributes are not perceived at the same time. Rather, the attribute that is processed fastest would be perceived first.
Psychophysical evidence for the existence of perceptual asynchronies is mixed, and a number of alternative explanations have been put forth. For the perception of motion and color, Moutoussis and Zeki (1997a, 1997b) have demonstrated that a color change tends to be perceived about $80 \mathrm{msec}$ before a change of motion direction. In one of their experiments, the perceptual asynchrony was determined by presenting red squares on a computer screen that oscillated up and down and changed color (red to green) at the same time, before, or after the change of motion direction. The participants had to judge which color corresponded to a particular motion direction. Moutoussis and Zeki (1997a, 1997b) found that when the change of motion direction preceded the color change by about $80 \mathrm{msec}$, participants perceived motion and color in synchrony.

\section{Changes of Direction Versus Changes of Position}

A subsequent study demonstrated that when color and motion changes appear in a nonrepetitive sequence or at slow alternation rate, the changes are perceived in synchrony when they are physically synchronous (Nishida \& Johnston, 2002). Nishida and Johnston suggested that the asynchrony could be the result of a noncorrespondence of temporal markers. More precisely, at high temporal frequencies, the visual system is unable to compare a firstorder change of stimulus color with a second-order change of stimulus motion, causing an asynchronous perception of color and motion. Second-order change refers to the fact that more than two samples are necessary in order to determine whether a change has occurred. For instance,

D. Kerzel, dirk.kerzel@pse.unige.ch 
in order to determine whether the direction of motion has changed, the initial and new direction of motion need to be determined by means of two differences among three successive positions. In contrast, a first-order change requires only one difference between two samples. At high frequencies, linking second-order motion changes to firstorder color changes becomes difficult and causes a perceptual asynchrony. According to Nishida and Johnston, however, this error is not due to differential latencies.

Somewhat at odds with the results of Nishida and Johnston (2002), the color-motion asynchrony has been found to persist with a single direction change (Linares \& LópezMoliner, 2006) and with two first-order changes (Aymoz \& Viviani, 2004; Viviani \& Aymoz, 2001). In Viviani and Aymoz's studies, participants had to judge whether a change of stimulus color occurred before or after motion onset. Detection of motion onset requires only one difference between two samples and is therefore a first-order change. According to Nishida and Johnston, no asynchrony between color and motion was expected, because similar change types have the same temporal limitations. However, Viviani and Aymoz confirmed an asynchrony with a single large square as the stimulus and with a single direction of motion (upward, to the right). It is interesting that the asynchrony vanished when biological motion stimuli were used (Aymoz \& Viviani, 2004). In addition, there is research suggesting that the task used (Adams \& Mamassian, 2004; Bedell, Chung, Ogmen, \& Patel, 2003), attention (Enns \& Oriet, 2004; Holcombe \& Cavanagh, 2006), and motion direction (Arnold \& Clifford, 2002) influence or explain the color-motion asynchrony.

\section{Abrupt Versus Continuous Changes}

Another inconsistency arises from the results of Cai and Schlag (2001). In their presently unpublished experiment, two moving bars moved in opposite directions and crossed each other at the screen center. At the same time, the bars' height decreased on each frame. In the middle of the trajectory, the bars changed their color for one frame (colored flash), and participants had to indicate the relative position of the change. The perceived position of the flash and the perceived height of the bar were displaced in the direction of motion. According to the authors, this suggests that abrupt changes (a flash) and continuous changes (displacement of the bar) are represented differentially in the brain, and an error occurs if the two representations are matched. In general, the results of Cai and Schlag's study are consistent with work on the flash-lag effect. In the flash-lag effect, a stationary flashed object (an abrupt event) appears to lag behind a continuously moving stimulus (Nijhawan, 1994). A number of explanations have been put forth to account for the flash-lag effect; however, there is no agreement on the mechanism underlying the illusion (e.g., Nijhawan, 2002). It is interesting that when the flash appears at the onset of the moving object (the flash-initiated cycle), the effect is as large as when the flash appears along the trajectory (Khurana \& Nijhawan, 1995). Moreover, Sheth, Nijhawan, and Shimojo (2000) demonstrated flash-lag effects with features other than object motion, such as color, luminance, spatial frequency, and entropy changes. In their experiments, one disk contained a feature that changed continuously, whereas a brief change of the respective feature was presented in a second disk. Participants perceived the feature flash as lagging the continuously changing feature. The illusion was also demonstrated at the onset of the continuously changing feature (flash-initiated cycle).

\section{Spatial Versus Temporal Errors}

Note that in Viviani and Aymoz's (2001; Aymoz \& Viviani, 2004) studies, the mismatch between color and motion was interpreted as being a temporal error: Color perception was found to precede motion perception. In contrast, only one among many explanations considers the flash-lag effect to be a temporal error (e.g., Purushothaman, Patel, Bedell, \& Ogmen, 1998): The latency of the moving object was supposed to be shorter than the latency of the flashed object. In general, it is difficult to disentangle spatial from temporal errors, because motion is defined as "displacement across the time." Consequently, there is ambiguity about what is actually measured in an experiment. Errors in the binding of color and motion could be of a spatial or temporal nature. In their first report of phenomenally asynchronous perception, Moutoussis and Zeki (1997a) considered the possibility of a spatial error and dismissed it:

We considered other explanations but found them implausible. Subjects may, for example, develop the strategy of reporting the colour at the end of each upward excursion, before the motion reverses. We tried to avoid situations like this by instructing subjects to bind each direction of motion to a colour, and each colour to a direction of motion, pay no attention to position, and not follow any particular square but rather look at the screen as a whole. (p. 396)

Although it is unclear whether position-based mechanisms can be ruled out by mere instruction, the quotation demonstrates the difficulty of separating space and time in motion perception (see also Kreegipuu \& Allik, 2003, 2004). Whether the observed errors were labeled as being temporal or spatial seemed to depend more on the research context than on the experimental paradigm: When comparing an abrupt change of color with the onset of motion, Viviani and Aymoz (2001) and Bedell et al. (2003) talked about temporal errors, because they framed their experiments in terms of Moutoussis and Zeki's (1997a, 1997b) work on asynchronous perception. In contrast, a comparison of a colored flash with the onset of feature motion in color space was not given a temporal interpretation (Sheth et al., 2000), because the study was motivated with respect to the flash-lag effect.

There are two consistent differences between studies on the flash-lag effect and studies on asynchronous perception: First, the abrupt change was transient (of type A-B-A) in the flash-lag studies and long lasting (type A-B) in the asynchronous perception studies. However, there is no reason to believe that the duration of the abrupt change would entail different processing mechanisms. Second, studies on the flash-lag effect used the same perceptual dimension in 
the abrupt and the continuous stimulus (mostly position) because they were interested in the differences between moving and stationary objects. The only notable exception is Cai and Schlag's (2001) study. In contrast, studies in the wake of Moutoussis and Zeki (1997a, 1997b) used more than a single perceptual dimension because they were interested in the temporal binding of perceptual features.

Inspection of Table 1 shows that as a general rule, abrupt events lag (either spatially or temporally) continuous events during ongoing motion and at motion onset. Although this is true for comparisons within a dimension (position, color, etc.), the situation is less clear for comparisons between dimensions. Whereas Cai and Schlag (2001) reported that the abrupt change lagged the continuous change during ongoing motion, Viviani and Aymoz (2001; Aymoz \& Viviani, 2004) reported the opposite error at motion onset. Yet another study found no differences between color and motion at motion onset (Bedell et al., 2003). Bedell et al. attributed the conflicting results to the large stimulus used in the experiments of Viviani and Aymoz. The large stimulus produced foveal color and peripheral motion information, so that matching the motion signals originating in the periphery to color signals originating in the fovea may have incurred a delay.

\section{Purpose of the Present Study}

There were three aims of the present study: First, Experiments 1 and 2 directly compared judgments about an abrupt change of color, with respect to a continuous change of position during ongoing motion and at motion onset. As outlined above, previous studies had measured either one or the other, and the results were inconsistent. In particular, we were interested in the differences that are due to the trajectory position. Although previous studies on the flash-lag effect have shown that similar errors occur at motion onset and along the trajectory, this does not seem to be the case when an abrupt color change and a continuous position change were compared. The present study aimed at validating and quantifying this difference.

We chose to describe our methods and results with respect to temporal parameters: perceptual asynchrony, perceived earlier, perceived later). This nomenclature re- flects our desire to simplify exposition, despite the fact that it is potentially wrong (see above). As outlined above, spatial errors may also play a role.

In the motion onset condition, we asked observers to indicate whether the color change occurred before the first displacement of the bars from the rest position (see Figure 1). In the along the trajectory condition, we asked observers to indicate whether the color change occurred before the bars passed the screen center (i.e., the rest position in the motion onset condition). Essentially, the only difference between the two conditions was the trajectory before the critical color change: In the motion onset condition, the bars were stationary; they were moving in the along the trajectory condition. Experiment 2 was similar to Experiment 1, except that we varied the velocity of object motion. It was possible that the detection of color changes would be shortened at high velocities (Kreegipuu, Murd, \& Allik, 2006).

Second, Experiments 3 through 5 were designed to replicate Viviani and Aymoz's (2001) study. Viviani and Aymoz used a large square as a stimulus that always moved diagonally, upward, to the right. In order to compare perceptual asynchronies at motion onset and along the trajectory, we used a different stimulus (two vertical bars). Because our results differed strongly from those of Viviani and Aymoz we ran three experiments, in which we successively approached their methods. In Experiment 3, we tested the role of cues arising from the relative motion of the two bars. In Experiment 4, we tested whether stimulus size had an influence on the color-motion asynchrony. In Experiment 5, we investigated whether predictable motion would induce shorter latencies of color in comparison with motion perception.

Third, Experiments 6 though 8 tested whether strategies, subjective isoluminance, or our material accounted for the failure to replicate the results from Viviani and Aymoz (2001). In Experiment 6, we slightly changed the experimental question in order to induce two different strategies. Observers were led to focus either on the period before motion onset or on the period after motion onset. In Experiment 7, we repeated some experimental conditions with 4 experienced observers and adjusted the stimuli to

Table 1

Summary of Previous Experiments

\begin{tabular}{|c|c|c|}
\hline \multirow[b]{2}{*}{ Abrupt Change } & \multicolumn{2}{|c|}{ Abrupt Change Perception } \\
\hline & Ongoing & Onset \\
\hline Position & $\begin{array}{l}\text { flashed position > object motion: } \\
\text { flash-lag effect (e.g., Nijhawan, 1994) }\end{array}$ & $\begin{array}{l}\text { flashed position }>\text { object motion: flash- } \\
\text { initiated cycle (e.g., Khurana \& Nijhawan, 1995) }\end{array}$ \\
\hline Feature & $\begin{array}{l}\text { flashed feature }>\text { feature motion } \\
\text { (Sheth et al., 2000) }\end{array}$ & $\begin{array}{l}\text { flashed feature }>\text { feature motion } \\
\text { (Sheth et al., 2000) }\end{array}$ \\
\hline \multirow[t]{2}{*}{ Color } & $\begin{array}{l}\text { color change }>\text { object motion } \\
(\text { Cai \& Schlag, 2001) }\end{array}$ & $\begin{array}{l}\text { color change }<\text { object motion } \\
\text { (Viviani \& Aymoz, 2001) }\end{array}$ \\
\hline & & $\begin{array}{l}\text { color change }=\text { object motion } \\
(\text { Bedell et al., 2003) }\end{array}$ \\
\hline
\end{tabular}

Note-The table indicates whether an abrupt change was perceived earlier $(<)$ or later $(>)$ than object/feature motion. The abrupt change was compared with the ongoing motion or with motion onset. Most studies indicate that the abrupt change is perceived after object/feature motion. The only exception is a study by Viviani and Aymoz (2001), in which color perception was reported to precede motion perception. Although the table describes the results in terms of a temporal error, an interpretation in terms of a spatial error is also possible. 
A

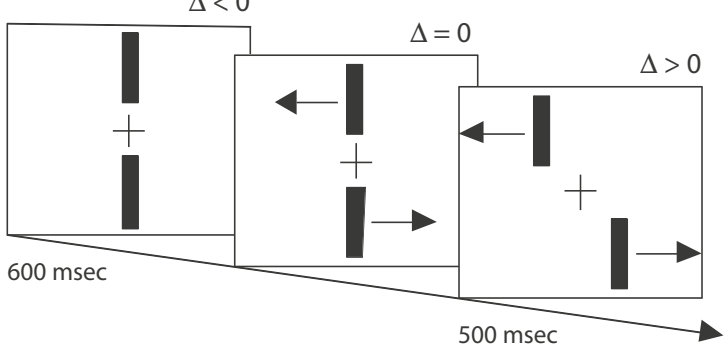

B

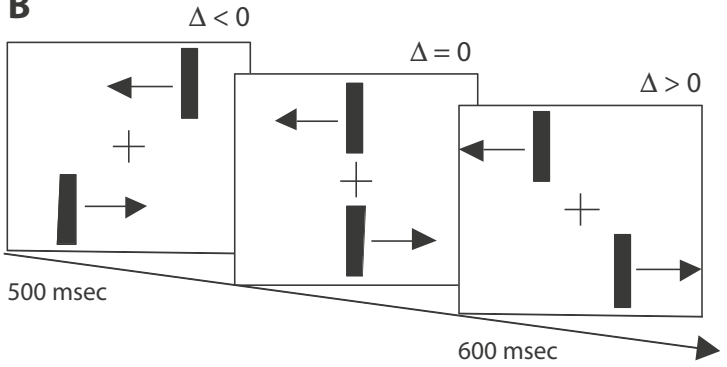

C

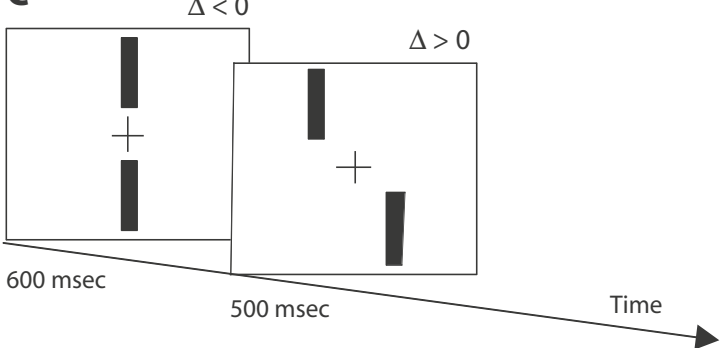

Figure 1. Overview of the experimental conditions. The stimulus onset asynchrony (SOA) is represented by the " $\Delta$ " symbol. (A) A trial in the motion onset condition. The bars are stationary at first, and then start to move. Negative SOAs indicate that the color change occurred before motion onset. (B) A trial in the along the trajectory condition. The bars move toward the center of the screen. Negative SOAs indicate that a color change occurred before the bars crossed the center of the screen. A trial in the control experiment (Experiment 8), in which 2 observers judged whether the color change preceded a step displacement. Negative SOAs indicate that the color change occurred when the bars were vertically aligned.

be isoluminant for each observer. Experiment 8 was designed to test our experimental apparatus and software.

\section{METHOD}

\section{Participants}

Students at the University of Geneva participated. They reported normal or corrected-to-normal visual acuity and were naive about the purpose of the experiment. Participants took part in only a single experiment, with the exception of Experiment 5, in which most of the students $(N=14)$ had participated in one of the previous experiments. Fourteen students participated in Experiment 1, 12 in Experiment 2, and 16 each in Experiments 3 through 5. Fourteen students participated in Experiment 6. Four experienced observers, including one of the authors, participated in Experiment 7. Both authors served as participants in Experiment 8 .

\section{Apparatus and Stimuli}

Stimuli were generated by a ViSaGe graphics adaptor (Cambridge Research System, Rochester, U.K.) and were presented on a 21-in. monitor at a resolution of $800 \times 600$ pixels at $160 \mathrm{~Hz}$. The luminance of the stimuli was $20.35 \mathrm{~cd} / \mathrm{m}^{2}$, with CIE (1930) chromatic coordinates of $x=0.621$ and $y=0.338$ for red and $x=0.282$ and $y=$ 0.608 for green. Only the physical luminance of the two colors was matched. Previous studies have either corrected for interindividual variation in isoluminance (e.g., Moutoussis \& Zeki, 1997a) or not (e.g., Aymoz \& Viviani, 2004; Moutoussis \& Zeki, 1997b; Viviani $\&$ Aymoz, 2001). Other studies have used multiples of detection threshold (Bedell et al., 2003). Across studies, results were not affected by whether strict isoluminance was assured or not; neither did it affect previous interpretations of the results. Because one of our aims was to replicate Viviani and Aymoz's study, we opted for physical, and not subjective, isoluminance. The background was gray, with a luminance of $54.34 \mathrm{~cd} / \mathrm{m}^{2}$. In most experiments, stimuli were colored bars $\left(0.34^{\circ} \times 4.85^{\circ}\right.$, horizontal $\times$ vertical, respectively $)$. When two bars were presented, they were shown above and below central fixation at an eccentricity of $2.4^{\circ}$ to the nearest edge. In some experiments, only a single colored object was shown: either a single bar $\left(0.34^{\circ} \times 4.85^{\circ}\right)$, or a large square $\left(9.07^{\circ} \times 9.07^{\circ}\right)$. The single object was shown at the vertical center of the screen. In conditions with two bars, a $1^{\circ} \times 1^{\circ}$ fixation cross was displayed at the center of the screen. Object motion was mostly horizontal, at a velocity of $15.36 \mathrm{deg} / \mathrm{sec}$. At the beginning of a trial, the object's color was always set to red. After a variable delay, it was changed to green.

The two main conditions were the motion onset and along-thetrajectory conditions (see Figure 1). In the motion onset condition, the object appeared at the horizontal screen center and was stationary for $600 \mathrm{msec}$. Then, it started to move for $500 \mathrm{msec}$. The color change occurred around the onset of object motion. Negative stimulus onset asynchronies (SOAs) indicate that the color change occurred before the first target displacement. Positive SOAs indicate that it occurred after the first target displacement.

In the along-the-trajectory condition, the two bars appeared at opposite eccentric locations and moved horizontally toward the screen center. For instance, one bar appeared in the upper right part of the screen and moved to the left while the other appeared in the lower left corner and moved to the right. The bars reached the horizontal screen center (the fixation cross) after $500 \mathrm{msec}$ and continued to move for another $600 \mathrm{msec}$. Negative SOAs indicate that the color change occurred before the bars crossed the horizontal screen center. Positive SOAs indicate that the color change occurred after they had passed the horizontal screen center.

Nine SOAs were selected according to the results of a pilot study. At motion onset, the SOAs were distributed symmetrically from -250 to $250 \mathrm{msec}$, in steps of $63 \mathrm{msec}$. Along the trajectory, SOAs were $-325,-225,-150,-100,-50,0,50,125$, and $225 \mathrm{msec}$.

\section{Procedure}

The experiments took place in a dimly lit room. Participants viewed the stimuli at a distance of $58 \mathrm{~cm}$ from the monitor. Head movements were restricted by a chinrest. In the motion onset condition, participants pressed one of two keys to indicate whether they perceived the object to change color before it started to move (color first) or perceived the motion onset before the color change (motion first).

In the along-the-trajectory condition, participants pressed one of two keys to indicate whether they perceived the objects to change color before they crossed the horizontal screen center (color first) or after they crossed it (motion first).

Experiments 1 and 2. Perceptual asynchronies were determined at motion onset and along the trajectory. The motion onset and along-the-trajectory conditions were blocked, and the block order was balanced across participants. The remaining conditions were shown in random order.

In Experiment 1, each of the nine SOAs were paired with each of two directions of motion; the upper bar moved to the left or right while the lower bar moved in the opposite direction. Each partici- 
pant worked through 540 trials: 2 (change condition) $\times 9(\mathrm{SOA}) \times$ 2 (direction) $\times 15$ (repetition).

Experiment 2 was set up the same as Experiment 1, with the exception that the velocity was randomly set to either 7.68 or $15.36 \mathrm{deg} / \mathrm{sec}$. Each participant worked through 1,152 trials: 2 (change condition) $\times$ 2 (velocity) $\times 9(\mathrm{SOA}) \times 2$ (direction) $\times 16$ (repetition). To prevent the effects of fatigue, the participants were tested in two sessions. The two change conditions were administered in both sessions.

Experiments 3 through 5. These experiments were designed to test whether the different stimulus parameters accounted for the discrepancy between our results from Experiments 1 and 2 and those from Viviani and Aymoz's (2001) study. Therefore, we ran different versions of the motion onset condition.

In Experiment 3, we reduced relative motion cues by displaying only a single bar at the screen center and removing the fixation cross (single bar condition). In the study byViviani and Aymoz (2001), only a single large square was shown. The two bars condition was the same as the motion onset condition of the previous experiments. Each participant worked through 576 trials -2 (number of bars) $\times$ $9(\mathrm{SOA}) \times 2$ (direction) $\times 16$ (repetition) -in random order.

In Experiment 4, the shape of the moving object was varied. Either a single bar or a single large square (as in Viviani \& Aymoz, 2001) was presented. No fixation cross was presented. Each participant worked through 576 trials -2 (object shape) $\times 9(\mathrm{SOA}) \times$ 2 (direction) $\times 16$ (repetition) - in random order.

In Experiment 5, effects of motion predictability were evaluated. A single moving square was presented. Instead of the horizontal trajectory used in the previous experiments, the target moved diagonally. In Viviani and Aymoz (2001; Aymoz \& Viviani, 2004), the target always moved to the upper right corner of the screen. In this experiment, the target moved from the center to either the upper right-hand or the lower left-hand corner at a velocity of $10.86 \mathrm{deg} /$ sec (compared with $10.76 \mathrm{deg} / \mathrm{sec}$ in Viviani \& Aymoz, 2001). In the predictable condition, the square always moved in the same direction, and the direction of motion was counterbalanced across participants. In the unpredictable condition, the direction of motion varied randomly from trial to trial. The predictable and unpredictable conditions were blocked, and the block order was balanced across participants. Each participant worked through 576 trials: 2 (predictability) $\times 9(\mathrm{SOA}) \times 2$ (direction) $\times 16$ (repetition).

Experiment 6. In Experiment 6, we investigated whether strategies influence perceptual judgments at motion onset. The large square and horizontal motion were presented. We instructed participants to focus either on the stationary phase of the stimulus and ignore the motion phase of the stimulus (focus stationary condition) or on the motion phase of the stimulus and to ignore the stationary phase of the stimulus (focus motion condition). The task was always a temporal-order judgment, but the question was put differently. Participants had to indicate whether there was a color change either when the stimulus was stationary (focus stationary) or when it was in motion (focus motion). Formally, these two questions are equivalent and should lead to similar responses. Subjectively, however, they may be different. Participants worked through 576 trials: 2 (strategy) $\times 9(\mathrm{SOA}) \times 2$ (direction) $\times 16$ (repetition). Strategies were blocked, and the block order was balanced across participants.

Experiment 7. In Experiment 7, points of subjective equality (PSEs) were determined for four experienced observers in the following three conditions: at motion onset with two bars, at motion onset with a square, and along the trajectory. The direction of motion was always unpredictable. Unlike in the previous experiment, we chose a staircase method to adjust the SOAs. The SOA decreased after each motion first response and increased after each color first response. This simple up-down staircase aims at $50 \%$ color first responses. About 300 trials were run for each condition and observer. The luminance of the two colors was matched for each observer individually, using a flicker technique (Cavanagh, MacLeod, \& Anstis, 1987).

Experiment 8. This experiment was a control to check for possible programming bugs or hardware failures. We repeated Experiments 1 and 2, but we replaced the motion by a step displacement at the SOA of zero. Initially, the bars were at the screen center and were shifted abruptly to another location (see Figure 1). The task was to indicate, by the participants' pressing of one of two response keys, whether the color change was perceived before or after the position change.

\section{RESULTS AND DISCUSSION}

In each experiment, the relative frequencies of the motion first responses of the participants were computed as a function of SOA and experimental condition. Data were collapsed across leftward and rightward motion. The logistic function was fit to the relative frequencies: $y=$ $1 /\{1+\exp [-(x-c) / a]\}$, where $a$ indicates the slope of the curve, $c$ estimates the PSE between color and motion, $x$ indicates the SOA, and $y$ represents the relative frequency of motion first responses. Negative PSE values indicate that the color change had to occur before the motion change in order for them to be perceived as being simultaneous, indicating that motion was perceived earlier than color. Conversely, positive values indicate that color was perceived earlier than motion, as claimed by previous studies. For the sake of clarity, we report PSE as a temporal measure, although both temporal and spatial processes may contribute. Furthermore, we also considered the slope value, $a$. Slope values provide a measure of the just noticeable difference. Note that - due to the exponential—large values of $a$ indicate shallow curves; that is, large slope values indicate a poor ability to discriminate.

Psychometric functions were calculated for each condition and participant (shown for Experiments 1 and 8 in Figure 2). The fit of individual functions was good $\left(R^{2}\right.$ of .89 to 1 ). To evaluate differences between conditions and differences from zero, $t$ tests were conducted on PSE and slope values. Results of $t$ tests comparing the means are presented in Table 2. We also calculated Pearson's correlation between the PSE values of the different conditions (see Table 2). Means and standard deviations are reported in the format $M \pm S D$. Means and standard deviations are in milliseconds.

\section{Experiments 1 and 2}

Because a two-way, within-participants ANOVA2 (change condition) $\times 2$ (velocity) - on the data of Experiment 2 did not reveal any effects involving velocity $(p>.7)$, we pooled the data of Experiments 1 and 2 . Mean PSEs are presented in the left part of Figure 3 and in Table 2. The negative PSEs indicate that an abrupt color change had to be presented before a continuous position change in order for them to be perceived as being synchronous. Thus, an abrupt color change was perceived later than a continuous position change. Along the trajectory, such a result is consistent with Cai and Schlag's (2001) abstract. Our $t$ tests confirmed that the lag of color along the trajectory was significantly different from zero $(-45 \pm 32)[t(25)=-7.12, p<.001]$. PSEs at motion onset were generally closer to zero, but were negative, indicating that color also lagged motion at motion onset. This result is opposite to what would be expected on the basis of Viviani and Aymoz's (2001) study, which reported 

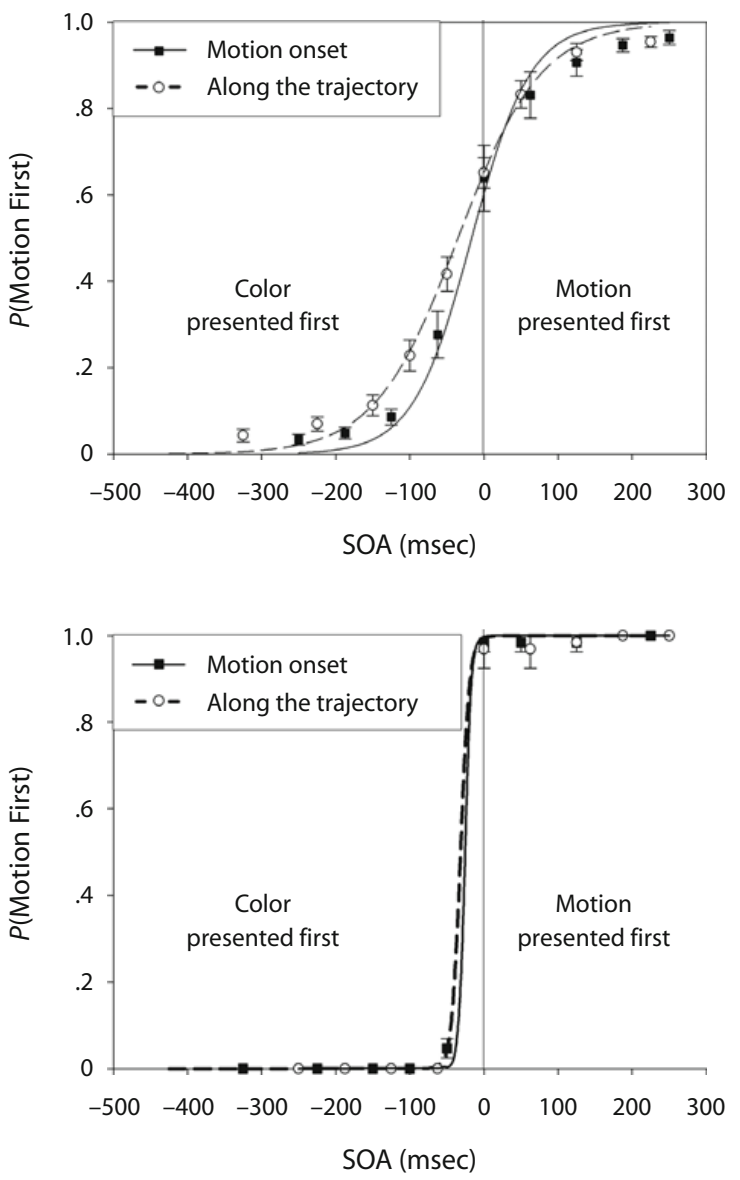

Figure 2. Mean probabilities of motion first responses and between-participants standard errors, as a function of the stimulus onset asynchrony (SOA) and experimental condition. The top panel shows data from Experiment 1, and the bottom panel shows data from the control experiment (Experiment 8). Negative SOAs indicate that the color change occurred before motion onset. In the control experiment, only a step function was shown (see text for explanation). Logistic functions were fit to the averaged data.

that color perception preceded motion perception. The negative PSE at motion onset was significantly different from zero $(-23 \pm 41)[t(25)=-2.81, p<.01]$. Furthermore, the difference between PSEs at motion onset and along the trajectory was significant $(22 \pm 46)[t(25)=$ $2.46, p<.05]$.

Slope values are plotted in the left part of Figure 4 . The larger slope values along the trajectory show that observers' ability to discriminate between the different SOAs was weak in comparison with those for the onset change condition. In other words, observers' judgments along the trajectory were less precise and more variable. The difference between slope values at motion onset and along the trajectory was significant $(17 \pm 21)[t(25)=4.1, p<$ $.001]$. The difference in precision between the two conditions is a first indication that the two tasks are solved differently or that they tap different perceptual mechanisms.
The scatterplot of individual PSE values in Figure 5 shows that there was no linear relationship between the two conditions (see also Table 2). The correlation was not significant $(r=.25, p=.22)$. If the two outliers apparent in the scatterplot are removed, the correlation drops to .08 . Because the correlation between the two conditions was low, it seems implausible that individual participants used the same strategy for solving the two tasks, or that the underlying mechanisms were the same. If this had been the case, individual PSEs should have been similar in the two conditions. The lack of correlation shows that this was not the case. As outlined above, judgments may be based on position along the trajectory and on temporal order at motion onset.

\section{Experiments 3 Through 5}

PSE values in Experiments 3 through 5 are graphed in the right part of Figure 3. Only the motion onset condition was run, and a number of different stimuli were used. Although PSEs were negative with one or two bars as stimuli, they tended to be positive with the large square that was also used by Viviani and Aymoz (2001). However, a significant asynchrony was only confirmed in the single bar condition of Experiment 3. As in Experiments 1 and 2 , the abrupt color change was perceived later than the continuous position change $(-22 \pm 32)[t(15)=-2.77$, $p<.05]$. This result is opposite to that of Viviani and Aymoz's study.

Furthermore, PSEs depended on object shape. In Experiment 3 , the abrupt color change was perceived earlier with two bars than with one $(-8 \pm 40 \mathrm{msec}$ vs. $-22 \pm$ $32 \mathrm{msec})[t(15)=2.69, p<.05]$. Remember that fast color perception is equivalent to large (positive) PSEs, and fast motion perception is equivalent to small (negative) PSEs. In Experiment 4, the abrupt color change was perceived earlier with a large square than with a bar $(10 \pm$ $48 \mathrm{msec}$ vs. $-6 \pm 47 \mathrm{msec})[t(15)=2.71, p<.05]$. Thus, larger stimuli (two bars vs. one bar, large square vs. one bar) reduced the negative PSEs and eventually even produced a slightly positive PSE. Thus, our results suggest that large objects make color perception faster.

Furthermore, correlations between the different onset change conditions were high and significant (see Table 2 and Figure 5). For each participant, PSE values in the two conditions were similar, which indicates that the two conditions tapped the same strategy or perceptual mechanism. This point is further corroborated by the slope values in the two conditions. The precision (or variability) of judgments did not differ between experimental conditions (see Figure 4), which is consistent with the idea that the various conditions were not fundamentally different.

\section{Experiment 6}

Neither strategy produced PSEs that were significantly different from zero (see Figure 3), and the two strategies did not differ from each other (see Table 2). Whereas preliminary ANOVAs (not reported) in all of the previous experiments had never shown effects that implied either order of task or order of stimulus presentation, task order significantly affected PSEs in the present experiment (see Figure 6). A two-way, mixed factors ANOVA-2 (strat- 
Table 2

\begin{tabular}{llccc}
\multicolumn{5}{c}{$\begin{array}{c}\text { Correlations and } \boldsymbol{t} \text { Tests Between Experimental Conditions in } \\
\text { Experiments 1 Through 6 }\end{array}$} \\
\hline \multicolumn{1}{c}{ Conditions } & $N$ & $t$ & $r$ \\
\hline Experiment & \multicolumn{1}{c}{$t$} & 26 & $2.46^{*}$ & .22 \\
1,2 & motion onset vs. along the trajectory & 16 & $2.69^{*}$ & $.85^{* *}$ \\
3 & one bar vs. two bars & 16 & $-2.71^{*}$ & $.89^{* *}$ \\
4 & bar vs. square & 16 & -0.16 & $.82^{* *}$ \\
5 & predictable vs. unpredictable & 14 & 0.05 & .24 \\
6 & focus stationary vs. focus motion &
\end{tabular}

Note-The mean points of subjective equality (PSE) of two experimental conditions were compared by $t$ test. Pearson's $r$ between individual PSEs in the two experimental conditions was calculated. ${ }^{*} p<.05 .{ }^{* *} p<.001$

egy) $\times 2$ (order) - revealed a significant interaction between order and strategy condition only $[F(1,12)=5.78$, $p<.05]$. When observers concentrated on the stimulus while it was stationary (focus stationary), PSEs were not different from zero, irrespective of whether the task was run in the first or second block $(-3 \pm 43$ vs. $-3 \pm 28)$. However, when observers were asked to focus on object motion, results were significantly affected by order. When the focus stationary strategy was induced in the first block, PSEs tended to be positive ( $33 \pm 59)$, and they tended to be negative $(-40 \pm 50)$ when it was induced in the second block. This difference was significant $[t(12)=2.46$, $p<.05$ ], suggesting that individual strategies may affect PSEs, albeit in a complicated way. Observers who adopt the focus motion strategy right away have a tendency to judge motion to have occurred before a change in color. In contrast, observers who change strategies in the course of an experiment will show the opposite trend. It is clear that strategies and their interactions will produce variable results, depending on which strategies observers adopt and on the order in which they are adopted. However, it seems unlikely that different strategies can account for the difference between Viviani and Aymoz's (2001) and our study. Collapsed across blocks, neither strategy produced positive PSEs.

Slope values did not differ significantly between the focus stationary and focus motion strategies $(58 \pm 30$ vs. $56 \pm 26)[t(13)=0.31, p=.76]$, suggesting that the strategy did not affect the ability to discriminate temporal order (see Figure 4). Moreover, there was no significant correlation (see Figure 5) between the two conditions ( $r=$ $.24, p=.40$ ). The lack of correlation confirms that we did indeed induce different strategies that produced uncorrelated results. Remember that Experiments 3 through 5 had always yielded significant correlations between two versions of the onset change condition. We succeeded in eliminating this consistency in the present experiment by inducing different strategies.

\section{Experiment 7}

PSEs were determined as before, with the following exception: When fitting the logistic function, the relative frequency of color first responses for a given SOA was weighted by the number of presentations of the respective SOA. All 4 participants tended to perceive the continuous position change before perceiving the abrupt color change (see Figure 7). This was true both along the trajectory and at motion onset. It is also clear from Figure 7 that the variability - even with trained observers - was considerable. B.U.'s asynchrony was largest, with two bars at motion onset. C.D. showed a larger asynchrony along the trajectory. D.K. showed the largest asynchrony, with a large square at motion onset. Finally, S.B. showed much larger asynchronies in the two motion onset conditions than along the trajectory. Although the present group of observers did not show a clear trend with regard to which condition had produced a larger asynchrony, all observers showed a negative asynchrony, indicating slower processing of the abrupt color change than of the continuous position change. This confirms that our failure to replicate faster color processing than motion processing is not due to the specific sample (untrained observers) used in Experiments 1 through 6 . Also, we adjusted the colors to be subjectively isoluminant. This did not affect the results and, in particular, did not produce positive PSEs.

\section{Experiment 8}

Figure 2 illustrates the psychometric curve, adjusted to the data of 2 observers. The observed step function is consistent with our expectations and shows that the apparatus and programs were working properly.

\section{Power Analysis}

In Experiments 4 through 6, we failed to replicate a temporal advantage of color processing over motion processing. Before concluding the effects reported by Viviani and Aymoz (2001) to be spurious, we conducted a power analysis using $\mathrm{G}^{*}$ Power 3 (Faul, Erdfelder, Lang, \& Buchner, 2007) to ensure we did not miss the effect due to lack of power. To this end, we pooled data from all conditions with our version of Viviani and Aymoz's large square $(N=$ 46). Viviani and Aymoz reported a mean asynchrony between color and motion of $57 \mathrm{msec}$, with a standard deviation of 32 (calculated from their Table 3 ). The effect size for matched pairs, $d_{z}$, is defined as the mean difference divided by its standard deviation. For Viviani and Aymoz's asynchrony, $d_{z}=1.80$, which is considered an extremely large effect. Cohen (1992) classified effect sizes of 0.2, 0.4 , and 0.8 as being small, medium, and large, respectively. An a priori power analysis suggests that only 5 observers are necessary for replicating such a large effect. A post hoc power analysis showed that with our sample size of 46 observers, and assuming a two-tailed $t$ test with $\alpha=.05$, the power to detect an effect of this size is 1 . The 


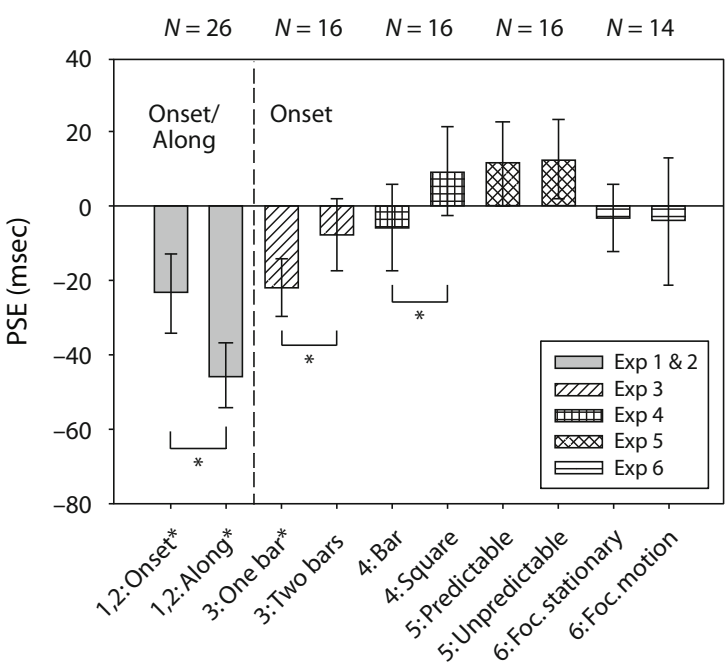

Figure 3. Mean PSEs (in milliseconds) and between-participants standard errors, as a function of experimental condition. The number preceding the condition title corresponds to the experiment number used in the text. Negative values indicate that participants perceived motion first (i.e., the color change had to be presented before the motion change in order to be perceived as being simultaneous). An asterisk in the condition title indicates that the mean was significantly different from zero ( $t$ test, $p<$ $.05)$. Significant differences between conditions are indicated by a square bracket and an asterisk $(t$ test, $p<.05)$.

power is defined as $1-\beta$, where $\beta$ is the probability of wrongly accepting $\mathrm{H}_{0}$, when in reality, $\mathrm{H}_{1}$ is true (Type II error). A power of 0.8 is considered an adequate compromise between the risk of a Type II error and expenses (Cohen, 1992). Consequently, we may be almost certain of not having incorrectly accepted $\mathrm{H}_{0}$, given the large power of our study. Actually, the probability of missing the effect $(\beta)$ was practically zero.

Nonetheless, one may object that the variability in our sample was larger than in Viviani and Aymoz (2001): The $S D$ was 32 in their sample of 20 observers and 43 in our sample of 46 observers, an increase of $130 \%$. Would it be possible to detect a true mean asynchrony of $53 \mathrm{msec}$, as in Viviani and Aymoz, with a variability of this magnitude? Sure; with a standard deviation of 43 and a sample size of 46, the smallest asynchrony that would have been significant is $13 \mathrm{msec}$ (critical $t$ value of 2.10 , two-tailed). However, we obtained a mean asynchrony of $7 \pm 43 \mathrm{msec}$, which is far from significant $[t(45)=1.10, p=.28]$.

\section{GENERAL DISCUSSION}

Along the trajectory, the abrupt color change was perceived about $40 \mathrm{msec}$ after the continuous position change. This replicates results reported in a conference abstract (Cai \& Schlag, 2001). At motion onset, the two changes were mostly perceived in synchrony, or color lagged motion slightly. Thus, the lag of color was smaller at motion onset than along the trajectory, suggesting that interdimensional asynchronies between color and motion differ between motion onset and trajectory. This is not the case for flashes and continuous motion defined along the same dimension (see Table 1). We believe that the differences we observed between the along the trajectory and motion onset conditions may be accounted for by task demands (see below).

It should be noted that although the between-participants variability was generally larger for judgments in the motion onset condition than in the along the trajectory condition, the individual discrimination performance, as measured by the slopes of the logistic functions, was actually better at motion onset than along the trajectory (see Figure 4). Thus, the mostly nonsignificant asynchronies in the motion onset condition cannot be due to poor discrimination of the color change. Rather, perceptual asynchronies were reliable within individual observers, but were overall close to zero.

Our results in the motion onset condition are in contradiction to a body of literature showing that if there is an asynchrony, color is perceived before motion (Aymoz \& Viviani, 2004; Moutoussis \& Zeki, 1997a, 1997b; Viviani \& Aymoz, 2001). To resolve this discrepancy, we successively approached previous methods. In fact, our large square condition is an almost exact replication of Viviani and Aymoz's experiment. However, we observed no significant positive asynchrony. When there was an asynchrony, color was actually perceived after motion (cf. the motion onset condition in Experiments 1 and 2 and the one bar condition in Experiment 3; see Figure 3). These results were confirmed with trained observers in Experiment 7. Moreover, we found some evidence that perceptual strategies (focus on stationary or moving period) affect PSEs (Experiment 6). However, there was no

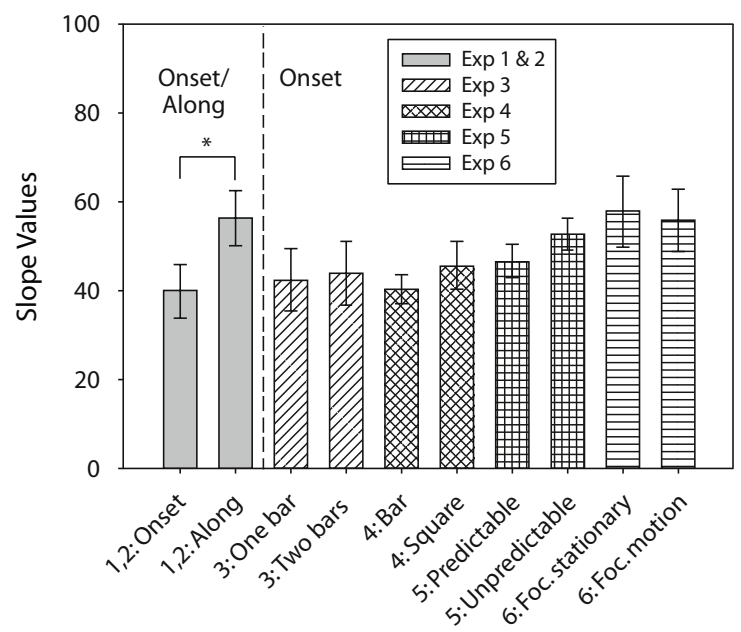

Figure 4. Slope values and between-participants standard errors, as a function of experimental condition. The number preceding the condition title corresponds to the experiment number used in the text. Higher values indicate shallow psychometric functions and weak discrimination performance. Significant differences between conditions are indicated by a square bracket and an asterisk ( $t$ test, $p<.05)$. 

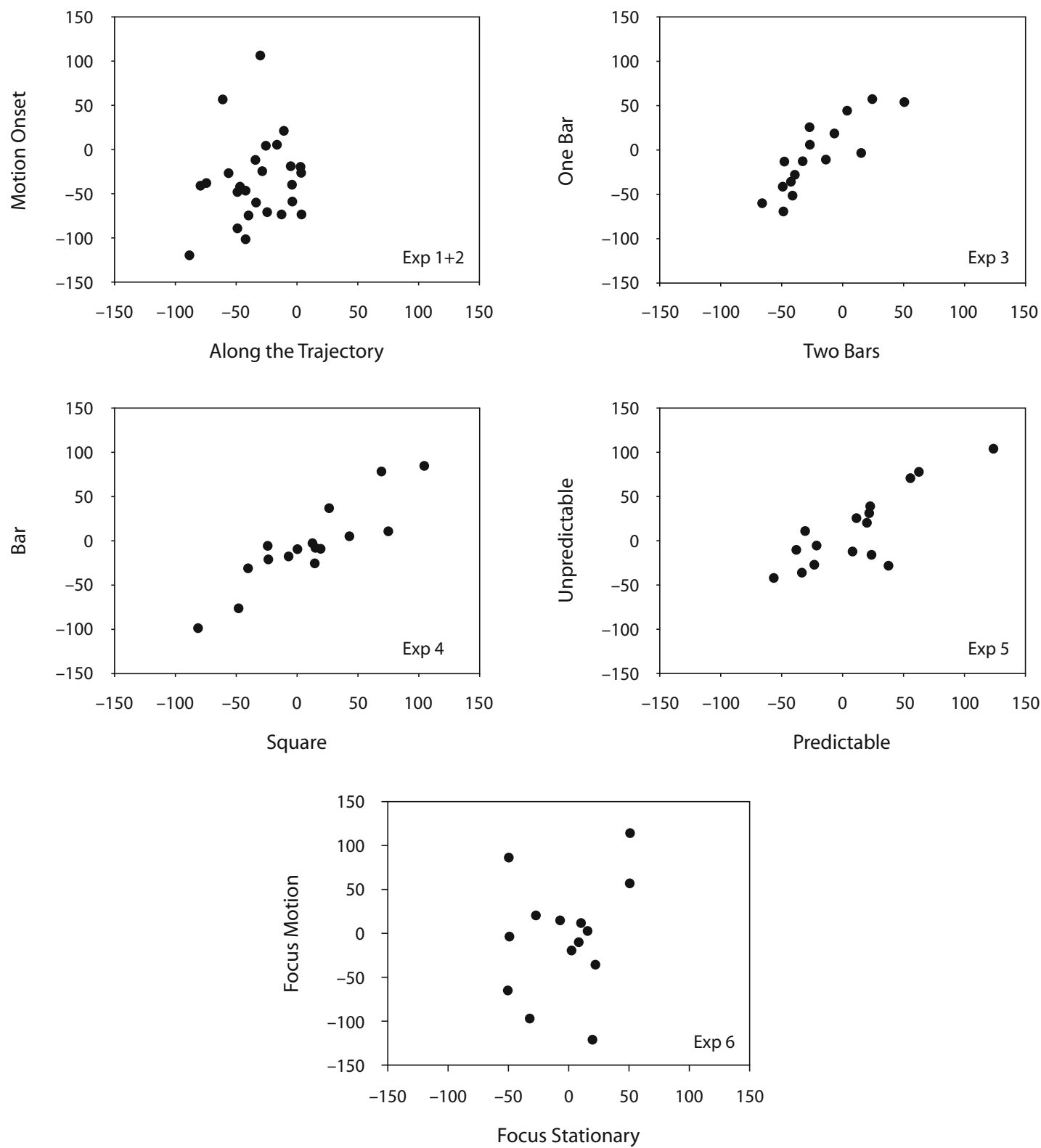

Figure 5. Scatterplots of individual points of subjective equality (PSEs, in milliseconds) in the respective experimental conditions of each experiment. Each point represents the PSEs of one participant. Correlations were significant in Experiments 3 through 5. Only the motion onset condition was run in Experiments 3 through 6.

simple relation between strategy and asynchrony. Rather, the effects of strategy were modulated by prior experience, and it is unlikely that the difference between this study and Viviani and Aymoz's can be accounted for by strategic effects.

On the basis of our results, we suggest that there is simply no reliable asynchrony between color and motion at motion onset. We therefore believe that the perceptual asynchrony between color and motion observed by Viviani and Aymoz (2001) was due to a sampling error. Actu- ally, recent experiments in Viviani and Aymoz's laboratory (Dunand, 2006) failed to reproduce the original result. In an unpublished thesis, Dunand reported a nonsignificant asynchrony of about $10 \mathrm{msec}$, indicating faster processing of color. These results are remarkably similar to ours (see rightmost bars in Figure 3). Furthermore, our results are fully consistent with those of recent studies that reported no significant asynchrony between color and motion, when two first-order changes had to be judged (Bedell et al., 2003; Nishida \& Johnston, 2002). If the null 


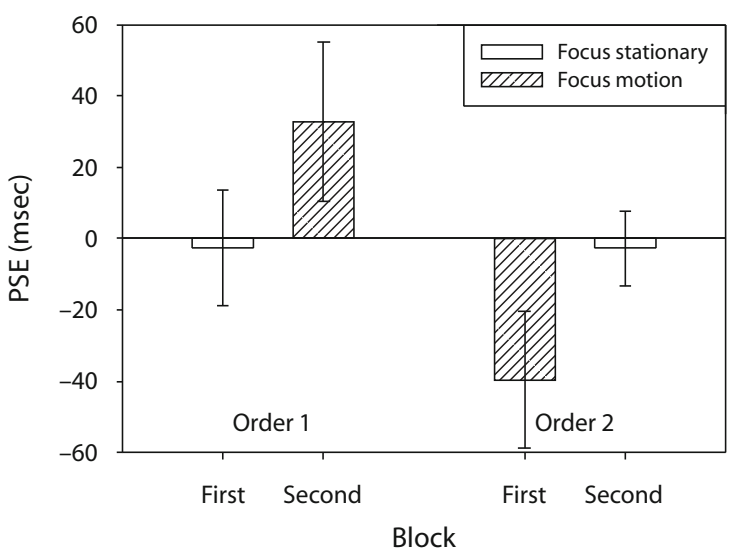

Figure 6. Results of Experiment 6. Mean PSEs (in milliseconds) and between-participants standard errors at motion onset in the focus stationary and focus motion conditions, as a function of block order. In the focus stationary condition, participants indicated whether they perceived a color change while the square was stationary. In the focus motion condition, participants indicated whether they perceived a color change while the square was moving.

hypothesis of no difference between the two conditions is true, occasional experiments may nonetheless confirm differences due to Type I errors in significance testing. Another possibility is a technical problem in Viviani and Aymoz's study: They used Adobe Authorware to present their stimuli. This software is very uncommon in psychophysical experiments, and it occurs to us that the accuracy of stimulus timing needs to be validated before we can trust results obtained with this software.

\section{Effects of Stimulus Size}

Furthermore, the results of Experiments 3 and 4 suggest that the lag of color relative to motion was reduced when the stimulus subtended a smaller area. The lag of the color change relative to motion onset was shorter with two bars than with one bar, and with a large square than with a single bar. This is consistent with the finding that color discrimination is better with large stimuli than with small stimuli (Abramov, Gordon, \& Chan, 1991). Here we show that larger stimuli make the perception of a color change not only better, but also faster (relative to motion). Thus, the large stimulus used by Viviani and Aymoz (2001; Aymoz \& Viviani, 2004) increased the processing speed of color relative to that of motion. Slower color perception with small stimuli may thus explain why color lagged motion in some experiments using bars, but never in experiments using large squares. However, effects of size cannot account for the discrepancy between Viviani and Aymoz's robust temporal advantage of color over motion and the zero asynchronies observed in our Experiments 4 through 6. Despite our efforts to approach their methods, we did not observe positive asynchronies with a large square. Our analyses show that this failure to replicate is not due to insufficient power, and Experiment 8 suggests that it is not due to technical problems, either.

Furthermore, we observed that stimulus velocity did not affect perceptual asynchronies in Experiment 2. This finding is consistent with Viviani and Aymoz (2001). In a previous article, reaction times to fast-moving objects were found to be reduced (Kreegipuu et al., 2006). It may be that reaction times are differentially affected by stimulus velocity, whereas relative judgments are not.

\section{The Role of Task Demands}

Finally, the results show that judgments about a color change at motion onset were not correlated with judgments about a color change along the trajectory (Experiments 1 and 2). In contrast, there was a strong correlation between judgments at motion onset (Experiments 3-5). A possible interpretation is that the lack of correlation reflects different underlying strategies or mechanisms at motion onset versus along the trajectory. Most likely, the two tasks involve position- and motion-based mechanisms to different degrees. Both tasks could be solved by evaluating the target position relative to a fixed spatial reference - that is, the fixation cross. According to this strategy, observers would judge whether the first occurrence of the color change was displaced relative to the fixation cross. Such a strategy was feasible at motion onset and along the trajectory. A second strategy was

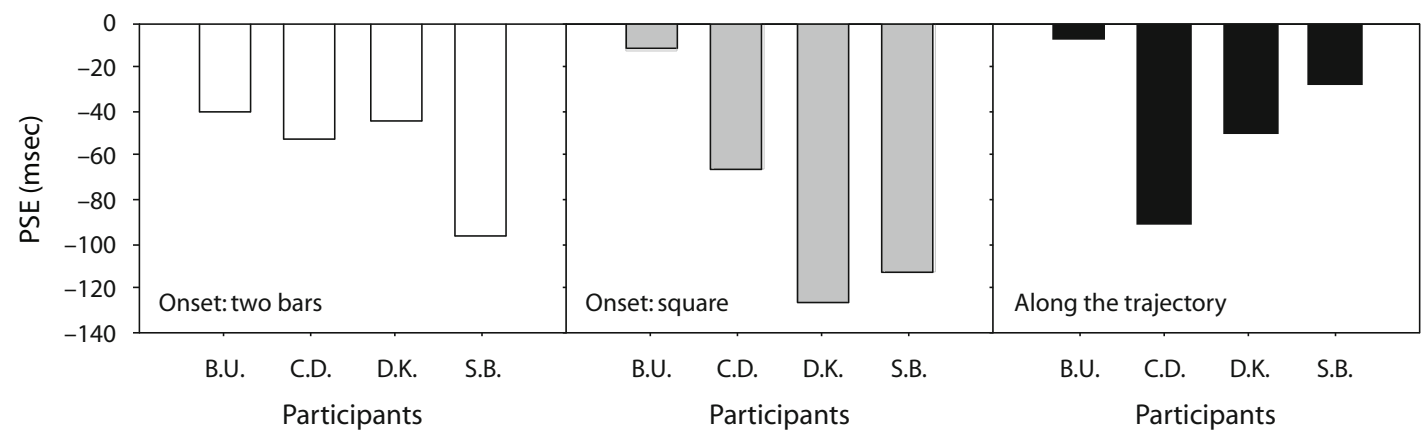

Figure 7. Results of Experiment 7. Mean PSEs (in milliseconds) for 4 participants, along the trajectory and at motion onset, with two bars and the large square. Negative values indicate that participants perceived the continuous position change first (i.e., the color change had to be presented before the motion change in order to be perceived as being simultaneous). 
possible at motion onset only: Observers could direct their attention to the temporal aspects of the situation and judge whether the occurrence of motion preceded the color change. Thus, only judgments at motion onset could use motion information, irrespective of position, and rely on temporal order. It may be that precise matching of two temporal events (motion onset and color change) is possible at motion onset, whereas a spatial error occurs along the trajectory. In other words, the mechanisms used to solve the task are temporal at motion onset and spatial along the trajectory, even if it is, in principle, possible to rely exclusively on position.

This account is partially in line with a recent study by Bedell et al. (2003). They demonstrated that color-motion asynchronies were different for judgments about the predominant color of squares moving in one specific direction (color correspondence task) and judgments about which change occurred first (temporal order task). Consistent with Moutoussis and Zeki (1997a), color tended to be perceived first in the color correspondence task, but no asynchrony was found in a temporal order judgment task (see also Johnston \& Nishida, 2001; Nishida \& Johnston, 2002) that included a condition in which motion onset was judged relative to a color change. Bedell et al. attributed the difference between tasks to the characteristics of the processing systems underlying judgments about transient events (i.e., changes of motion direction or motion onset) and of a system underlying judgments about sustained changes (i.e., the attributes of oscillating stimuli). The latter system integrated changes over larger temporal intervals. According to the model, transient information would be available earlier, and it would be less influenced by different latencies of spatiotemporal integration.

It is not entirely clear whether judgments of position would be treated by the sustained system, but it is clear that some spatiotemporal integration is necessary for perceiving the position of moving stimuli (Eagleman \& Sejnowski, 2000). If one were to subsume position judgments under the sustained system and the abrupt color change under the transient system, the lag of color relative to motion in our along the trajectory condition would indicate slower processing of transient relative to sustained processing components. This conclusion is opposite to Bedell et al.'s (2003), but is fully consistent with Cai and Schlag's (2001). However, although it is tempting to match "abrupt versus continuous" (Cai \& Schlag, 2001) to "transient versus sustained" (Bedell et al., 2003), the notions have not been sufficiently worked out to clearly make this link. In particular, Bedell et al.'s model only comprises color correspondence and temporal order judgment tasks, and relative position tasks have not been discussed. As task interacts with processing system, predictions for the along the trajectory condition are difficult to derive from the model.

\section{The Role of Eccentricity}

Furthermore, Bedell et al. (2003) attributed the asynchronous perception of color and motion reported by Viviani and Aymoz (2001; Aymoz \& Viviani, 2004) to the different eccentricities of color and motion stimuli in their study. The large stimulus in Viviani and Aymoz's studies produced foveal color and peripheral motion information. The lag of motion may therefore be due to the additional time required for comparing foveal color and peripheral motion signals. However, our experiments show that differences between foveal and eccentric processing cannot account for the discrepancy: Asynchronies between color and motion were absent with peripheral motion and foveal color stimuli (large square, Experiments 4-6), but also with peripheral objects (bars, Experiments 3 and 4) that provided combined color and motion signals in the periphery. However, we observed that the asynchrony between the abrupt color change and the continuous position change differed as a function of stimulus size. Larger stimuli (two bars, or the single large square) speeded up the processing of color changes.

\section{An Account in Terms of Differential Latencies}

In a seminal article, Metzger (1932) suggested that latencies of motion perception are longest at motion onset and decrease as motion progresses. His demonstration of the flash-lag effect provided evidence for this idea: Metzger conceived of a flash as being the first position of a moving object. Because latencies are longest at the onset of motion, the flash is expected to perceptually lag the moving object. Metzger's account may be applied to our results. If the latency of color perception does not change, motion is perceived simultaneously with color at motion onset, because latencies for motion perception are longest. Along the trajectory, latencies of motion perception decrease, and motion is perceived earlier than color. However, Metzger's differential latency account cannot explain misalignment in the flash-initiated cycle, because the flash should have the same latency as the first position of the moving object and should, therefore, be perceived as being aligned with the first position of the moving object.

In summary, perceptual asynchronies seem to be influenced by a large number of task and stimulus characteristics, as well as their interactions, which makes precise predictions very difficult. However, it seems unlikely that color precedes motion processing at motion onset in a consistent manner. In contrast, color lags motion consistently (temporally or spatially) when the color change occurs along the trajectory. Thus, the trajectory position affects the order of perceived events (or the spatial error) when the abrupt and continuous events are defined along different dimensions (color and position). This is not the case for motion and flashed stimuli defined along the same dimension.

\section{AUTHOR NOTE}

The authors were supported by Swiss National Foundation Grant 10011-107768/1. Correspondence concerning this article should be addressed to D. Kerzel, Faculté de Psychologie et des Sciences de l'Éducation, Université de Genève, 40 Boulevard du Pont d'Arve, CH-1205 Genève, Switzerland (e-mail: dirk.kerzel@pse.unige.ch).

\section{REFERENCES}

Abramov, I., Gordon, J., \& Chan, H. (1991). Color appearance in the peripheral retina: Effects of stimulus size. Journal of the Optical Society of America A, 8, 404-414 
Adams, W. J., \& Mamassian, P. (2004). The effects of task and saliency on latencies for colour and motion processing. Proceedings of the Royal Society B, 271, 139-146.

Arnold, D. H., \& Clifford, C. W. G. (2002). Determinants of asynchronous processing in vision. Proceedings of the Royal Society B, 269, 579-583.

Aymoz, C., \& ViViani, P. (2004). Perceptual asynchronies for biological and non-biological visual events. Vision Research, 44, 1547-1563.

Bedell, H. E., Chung, S. T. L., Ogmen, H., \& Patel, S. S. (2003). Color and motion: Which is the tortoise and which is the hare? Vision Research, 43, 2403-2412.

CAI, R., \& SchlaG, J. (2001). A new form of illusory conjunction between color and shape [Abstract]. Journal of Vision, 1(3), 127a.

Cavanagh, P., MacLeod, D. I. A., \& Anstis, S. M. (1987). Equiluminance: Spatial and temporal factors and the contribution of bluesensitive cones. Journal of the Optical Society of America A, 4, 1428-1438.

Cohen, J. (1992). A power primer. Psychological Bulletin, 112, 155-159.

DunAND, C. (2006). Influence du phénomène de causalité sur l'asynchronie dans la perception de la couleur et du mouvement. Unpublished doctoral dissertation, University of Geneva, Geneva, Switzerland.

Eagleman, D. M., \& SeJnowski, T. J. (2000). Motion integration and postdiction in visual awareness. Science, 287, 2036-2038.

Engel, A. K., Fries, P., König, P., Brecht, M., \& Singer, W. (1999). Temporal binding, binocular rivalry, and consciousness. Consciousness \& Cognition, 8, 128-151.

ENNS, J. T., \& ORIET, C. (2004). Perceptual asynchrony: Modularity of consciousness or object updating? [Abstract]. Journal of Vision, 4(8), $27 \mathrm{a}$

Faul, F., Erdfelder, E., Lang, A.-G., \& Buchner, A. (2007) $G^{*}$ Power 3: A flexible statistical power analysis program for the social, behavioral, and biomedical sciences. Behavior Research Methods, 39, 175-191.

Holcombe, A. O., \& Cavanagh, P. (2006). Apparent asynchrony between the perception of color and motion: An issue of different latencies or of attention? [Abstract]. Journal of Vision, 6(6), 1004a.

Johnston, A., \& NishidA, S. (2001). Time perception: Brain time or event time? Current Biology, 11, R427-R430.

Khurana, B., \& NiJHaWAn, R. (1995). Extrapolation or attention shift. Nature, 378, 566.
KreegipuU, K., \& AlliK, J. (2003). Perceived onset time and position of a moving stimulus. Vision Research, 43, 1625-1635.

KreegipuU, K., \& Allik, J. (2004). Confusion of space and time in the flash-lag effect. Perception, 33, 293-306.

KreegipuU, K., Murd, C., \& Allik, J. (2006). Detection of colour changes in a moving object. Vision Research, 46, 1848-1855.

Linares, D., \& LóPeZ-Moliner, J. (2006). Perceptual asynchrony between color and motion with a single direction change. Journal of Vision, 6, 974-981.

Livingstone, M., \& Hubel, D. (1988). Segregation of form, color, movement, and depth: Anatomy, physiology, and perception. Science, 240, 740-749.

Metzger, W. (1932). Versuch einer gemeinsamen Theorie der Phänomene Fröhlichs und Hazelhoffs und Kritik ihrer Verfahren zur Messung der Empfindungszeit [An attempt at a common theory of Fröhlich's and Hazelhoff's phenomena and a critique of their procedure for measuring sensation time]. Psychologische Forschung, 16, 176-200.

Moutoussis, K., \& ZEKI, S. (1997a). A direct demonstration of perceptual asynchrony in vision. Proceedings of the Royal Society B, 264, 393-399.

Moutoussis, K., \& ZEKI, S. (1997b). Functional segregation and temporal hierarchy of the visual perceptive systems. Proceedings of the Royal Society B, 264, 1407-1414.

NiJHawan, R. (1994). Motion extrapolation in catching. Nature, 370, 256-257.

NiJHaWAn, R. (2002). Neural delays, visual motion and the flash-lag effect. Trends in Cognitive Sciences, 6, 387.

Nishida, S., \& Johnston, A. (2002). Marker correspondence, not processing latency, determines temporal binding of visual attributes. Current Biology, 12, 359-368.

Purushothaman, G., Patel, S. S., Bedell, H. E., \& Ogmen, H. (1998). Moving ahead through differential visual latency. Nature, 396, 424.

Sheth, B. R., Nijhawan, R., \& Shimojo, S. (2000). Changing objects lead briefly flashed ones. Nature Neuroscience, 3, 489-495.

Treisman, A. (1999). Solutions to the binding problem: Progress through controversy and convergence. Neuron, 24, 105-110.

Viviani, P., \& Aymoz, C. (2001). Colour, form, and movement are not perceived simultaneously. Vision Research, 41, 2909-2918.

(Manuscript received April 3, 2007; revision accepted for publication March 13, 2008.) 